\title{
Direct Brachial Approach for Acute Basilar Artery Occlusion: Technical Note and Preliminary Clinical Experience
}

\author{
Teishiki Shibata, $\mathrm{MD}^{1}$, Masahiro Oomura, $\mathrm{MD}, \mathrm{PhD}^{2}$, Yusuke Nishikawa, $\mathrm{MD}, \mathrm{PhD}^{1}$, \\ Mitsuhito Mase, $\mathrm{MD}, \mathrm{PhD}^{1}$ \\ ${ }^{1}$ Department of Neurosurgery, Nagoya City University Graduate School of Medical Sciences, Nagoya, Japan \\ ${ }^{2}$ Department of Neurology, Nagoya City University Graduate School of Medical Sciences, Nagoya, Japan
}

\begin{abstract}
In mechanical endovascular thrombectomy (MET) for acute basilar artery occlusion (ABAO) in the elderly, navigating a guide catheter via the femoral artery may be difficult due to the approach route's significant tortuosity. To resolve this problem, we have been performing a technique that uses a direct brachial approach (DiBA) with a large-bore aspiration catheter. We reported our preliminary clinical experience with this technique. MET for ABAO using the DiBA technique was performed on 4 consecutive patients between August 2017 and December 2018. In all patients, thrombolysis in cerebral infarction 2B or 3 recanalization was achieved, but the modified Rankin Scale at 90 days was $\geq 4$. There were no technical difficulties or complications with this technique. The DiBA technique is an effective and feasible approach in MET for ABAO. Although excellent clinical outcomes could not be achieved, the angiographic outcomes were excellent with no technical complications. This approach can be an alternative to the femoral artery approach, particularly for tortuous arteries in the elderly.
\end{abstract}

Key Words: Stroke; Basilar artery; Brachial artery; Thrombectomy; Endovascular procedures

\section{INTRODUCTION}

Recently, mechanical endovascular thrombectomy (MET)'s efficacy for acute ischemic stroke (AIS) with large vessel occlusion (LVO) in the anterior circulation has been demonstrated. ${ }^{1-5}$ Moreover, earlier reperfusion has been associated with lower degrees of disability. ${ }^{6}$ In the posterior circulation, the efficacy of MET for AIS remains uncertain; however, it has been suggested that earlier reperfusion is important for a good outcome. Although MET is frequently performed in the elderly, placement of a guide catheter via a transfemoral route, partic- ularly into the posterior circulation, may be difficult due to the significant tortuosity of arteries ${ }^{8}$ and may delay reperfusion. The transbrachial approach is an alternative technique for the posterior circulation ${ }^{9}$ because the brachial artery is near the ipsilateral vertebral artery (VA), making manipulation of catheter easier.

To shorten the time for guide catheter placement during MET for acute basilar artery occlusion (ABAO), all METs for $A B A O$ have been performed via the transbrachial approach with a largebore aspiration catheter without using a guide catheter since August 2017. In this technique, which we called the direct

\author{
Correspondence to: \\ Teishiki Shibata, MD \\ Department of Neurosurgery, Na- \\ goya City University Graduate School \\ of Medical Sciences, 1 Kawasumi, \\ Mizuho-cho, Mizuho-ku, Nagoya 467- \\ 8601, Japan \\ Tel: +81-52-853-8286 \\ Fax: +81-52-851-5541 \\ E-mail: tteishikii@gmail.com
}

Received: December 20, 2019

Revised: December 26, 2019

Accepted: December 30, 2019

Copyright $\odot 2020$ Korean Society of Interventional Neuroradiology

This is an Open Access article distributed under the terms of the Creative Commons Attribution Non-Commercial License (http://creativecommons.org/licenses/by-nc/3.0) which permits unrestricted non-commercial use, distribution, and reproduction in any medium, provided the original work is properly cited.

pISSN 2093-9043 eISSN 2233-6273 
brachial approach (DiBA), we used the Penumbra 5MAX ACE reperfusion catheter (Penumbra Inc., Alameda, CA, USA) as a guide catheter. Herein, we reported our preliminary clinical experience with this technique.

\section{MATERIALS AND METHODS}

We retrospectively analyzed the data of 4 consecutive pa- tients who underwent MET for ABAO using the DiBA technique between August 2017 and December 2018. Patient characteristics, endovascular procedure details, technical complications, and angiographic and clinical outcomes were analyzed. Particularly, the technical complications of the transbrachial approach included spasm, occlusion, and pseudoaneurysm of the brachial artery and median nerve palsy. The outcome variables of interest included puncture to guide (PTG) time, which was defined as the duration from
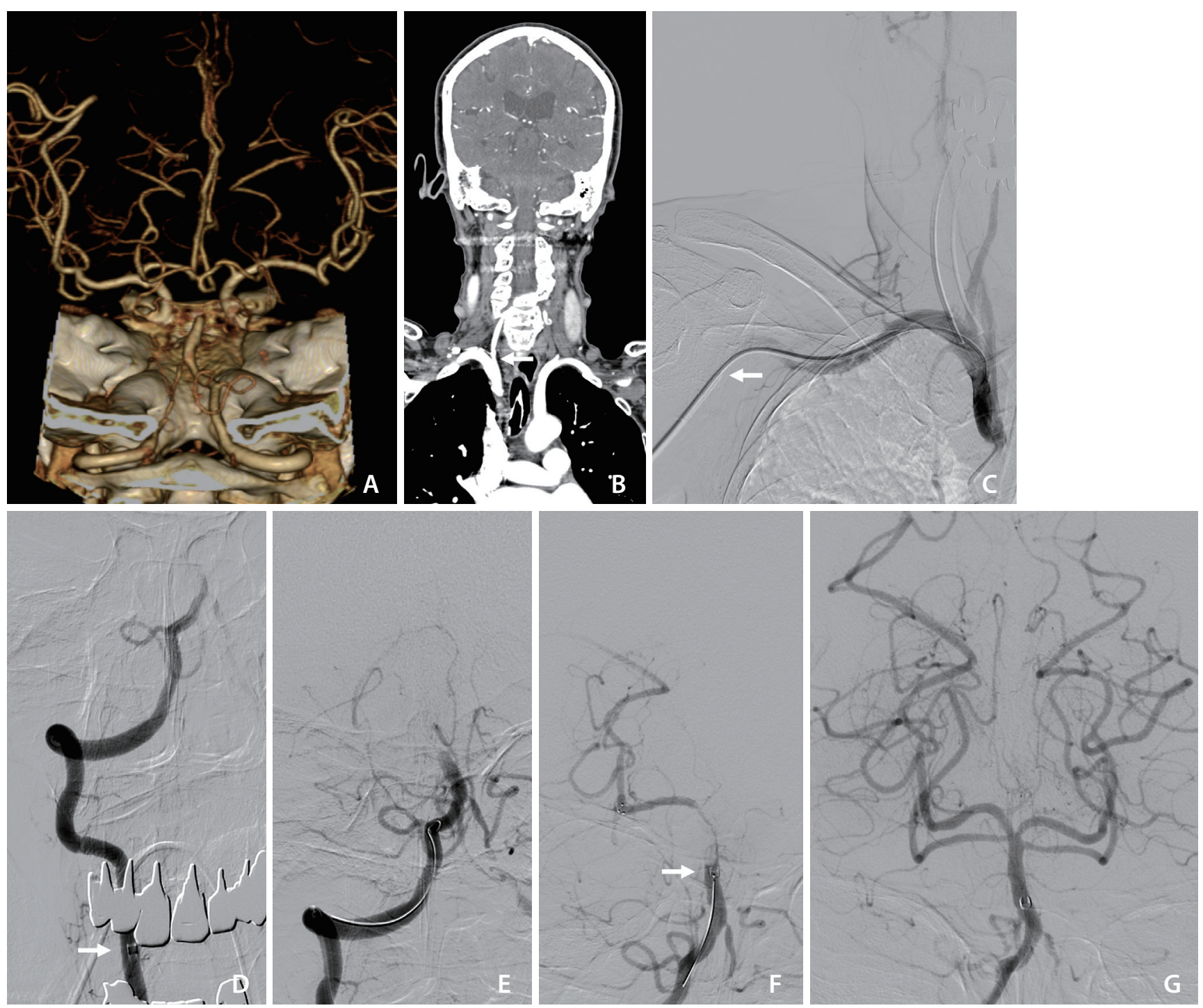

Fig. 1. The DiBA technique for an $A B A O$. CTA shows an $A B A O(\mathbf{A})$ and the assessment of the access route including the right VA ostium (B, arrow). (C) A 6-F introducer sheath measuring $25 \mathrm{~cm}$ is inserted in the right brachial artery (arrow), and the 5MAX ACE (Penumbra Inc., Alameda, CA, USA) is navigated using a 0.035 -inch guidewire or a catheter exchange technique. The 5MAX ACE is positioned at the VA V2 position (D, arrow), and a microcatheter and a microwire are navigated (E) to cross the thrombus. (F) After deployment of the stent retriever to the site of occlusion and positioning of the 5MAX ACE to the proximal site of the occlusion (arrow), immediate flow restoration was observed. (G) Finally, $\mathrm{TICl} 3$ recanalization was achieved. DiBA, direct brachial approach; ABAO, acute basilar artery occlusion; CTA, computed tomography angiography; VA, vertebral artery; TICI, thrombolysis in cerebral infarction. 
puncture to guide catheter placement in the appropriate position, and puncture to recanalization (PTR) time. The other outcomes analyzed included thrombolysis in cerebral infarction $(\mathrm{TICl})$ grade, score on the modified Rankin Scale (mRS) at 90 days, death due to any cause, and symptomatic intracranial hemorrhage, which was defined as an exacerbation based on the National Institutes of Health Stroke Scale score. All procedures were performed by board-certified neurosurgeons (T.S., Y.N., and M.M.) or an interventional neurologist (M.O.) of the Japanese Society of Neuroendovascular Therapy. The Institutional Review Board approval was obtained at the Nagoya City University Graduate School of Medical Sciences in Nagoya, Japan.

\section{The DiBA technique}

On admission, the patients underwent brain computed tomography (CT) and CT angiography (CTA). CTA was performed from the aortic arch to the cerebral vertex to assess the presence of LVO and the access route in case MET was necessary. If AIS in the posterior circulation was confirmed, brain magnetic resonance imaging was performed to exclude any large brainstem infarction. Subsequently, the DiBA technique was performed (Fig. 1).

First, the diameter of the bilateral VA was confirmed on CTA. MET was performed via the right brachial artery as long as the diameter of the right VA was large enough to accommodate the 5MAX ACE, irrespective of dominancy. If the right VA was hypoplastic or aplastic, MET was performed via the left brachial artery. A 6-F introducer sheath measuring $25 \mathrm{~cm}$ was inserted in a brachial artery. The $5 \mathrm{MAX}$ ACE was directly inserted into this introducer sheath with a 0.035 -inch guidewire, and navigated from the subclavian artery to the VA at the V2 position. Alternatively, a 4-F catheter was positioned at the VA, and then the 5MAX ACE was navigated into the VA using a catheter exchange technique. A combination of stent retriever (Solitaire FR, Medtronic Inc., Minneapolis, MN, USA) and aspiration techniques were performed..$^{10}$ Under roadmap guidance, the microcatheter and microwire that the operator chose were navigated past the thrombus; the most commonly used were the Marksman microcatheter (Medtronic Inc.) and the 0.014-inch ASAHI CHIKAI guidewire (ASAHI INTEC CO., Aichi, Japan). At the same time, the 5MAX ACE was delivered and positioned just proximal to the thrombus. If this was difficult, the 5MAX ACE was delivered after deploying the stent retriever, which was used as an anchor. After deploying the stent retriever to the

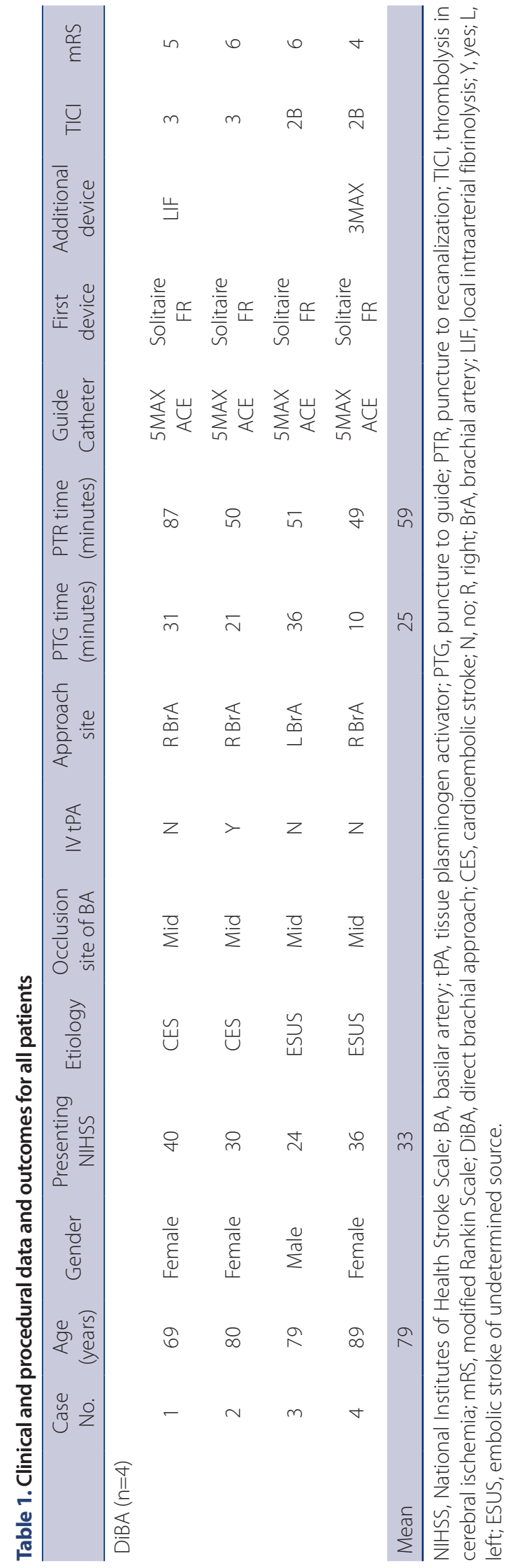


site of occlusion, the microcatheter was removed and the stent retriever was withdrawn into the $5 \mathrm{MAX} A C E$, which was aspirated with the Penumbra aspiration pump. After the MET, hemostasis at the brachial puncture site was achieved by using manual compression.

\section{RESULTS}

Four patients were included in our study. The patient characteristics are summarized in Table 1. In only 1 out of 4 patients, MET was performed via the left brachial artery because the right VA was hypoplastic (case 3). The mean PTG and PTR times were 25 minutes (range, 10-36) and 59 minutes (range, 49-87), respectively. Although in case 3, MET was performed via the left brachial artery, the PTG and PTR times did not significantly differ compared with the other 3 patients. For all patients, MET achieved TICI 2B or 3 recanalization, but the $\mathrm{mRS}$ at 90 days was $\geq 4$. There was no symptomatic intracranial hemorrhage, but there were 2 deaths; 1 patient died because of large brainstem infarction (case 2), and the other developed $A B A O$ during hospitalization for pneumonia and died due to worsening pneumonia (case 3). In all procedures, vasodilators were not used and no technical complications occurred, including complications of the transbrachial approach.

\section{DISCUSSION}

In this study, we analyzed the efficacy and feasibility of the DiBA technique in patients with $\mathrm{ABAO}$. In all patients, $\mathrm{TICl} 2 \mathrm{~B}$ or 3 recanalization was achieved and there were no technical difficulties or complications. The PTR time has been recommended to be within 30 minutes; ${ }^{11}$ however, in this study, we averaged 59 minutes before reperfusion was obtained. The longer PTR time was considered to be attributable to the operator skills and patients' high age rather than to the procedure itself. Therefore, improvement of operator skills would be necessary for further reduction in time to reperfusion. Additionally, a recent study has reported that in MET for ABAO, the rate of poor outcome (mRS, 4-6) was $45.8 \% .^{12}$ In our study, all patients had poor outcomes. However, it was thought that older patients with a high National Institutes of Health Stroke Scale (NIHSS) were associated with poor outcomes, as has been previously reported. ${ }^{12}$
In an era of MET where effective devices are available, the time from puncture to placement of a guide catheter should be crucial to achieving a rapid recanalization. Anatomical configuration can cause difficulty in manipulation of catheters. It is quite apparent that placement of a guide catheter to the right VA through a type III aorta via the femoral artery is more difficult compared with an approach via the ipsilateral brachial artery. In this study, the DiBA technique was successfully used to navigate a guide catheter to a targeted vessel in all 4 patients. Lee et al. ${ }^{13}$ reported their clinical experience of neurointerventional procedures via the transbrachial and transradial approaches. They mentioned the superiority of transbrachial or transradial approach to bypass the tortuosity of the aorta and the iliac artery. ${ }^{13}$

In 2018, Ishikawa et al. ${ }^{14}$ previously reported a technique that was similar to the DiBA technique. In that approach, ADAPT (a direct aspiration first pass technique) ${ }^{15}$ was initially performed on both the anterior and posterior circulation. On the other hand, the DiBA technique initially used a combination of stent retriever and aspiration for the ABAO; therefore, replacing the 5 MAX ACE via the brachial artery would not be necessary in case of MET failure.

Using a balloon guide catheter (BGC) with a stent retriever had been reported to improve revascularization and clinical outcomes. ${ }^{16}$ However, in MET for ABAO, BGC placement is often difficult because of the small caliber of the VA. Moreover, a BGC would not be useful for proximal flow control when the contralateral VA is patent. With the DiBA technique, the stent retriever can be withdrawn into the SMAX ACE that is placed at a basilar artery and aspirated, thereby preventing embolization to new territories (ENT). ${ }^{10}$

For this reason, to prevent ENT, the use of the Penumbra system is recommended during MET for ABAO. In case of using both a guide catheter and the 5MAX ACE, an 8-F or larger guide catheter (or a 6-F or larger guide sheath) is needed, but such a large profile guide catheter can be wedged in the VA. When the wedge of the VA and flow restriction occur, brainstem infarction in patients with hypoplasia of the contralateral VA or spinal cord infarction may occur. ${ }^{17}$ The DiBA technique is considered to reduce these complications because it does not use a large-bore catheter.

Atherosclerotic plaque is abundant in the infrarenal abdominal arota, iliac and femoral bifurcations, and carotid bifurcations; the routes from radial or brachial artery are considered to elicit less mechanical irritations of the aforementioned atherosclerotic plaques compared with the 
transfemoral route, resulting in a lower risk of cholesterol embolization. ${ }^{18,19}$ Particularly in the elderly, not only significant tortuosity but also atherosclerotic changes of arteries are frequently observed. Therefore, the DiBA technique should be useful for the elderly to reduce the incidence of cholesterol embolization, although it is infrequently encountered.

Iwata et al. ${ }^{9}$ reported that a transbrachial approach using a 6-F guide catheter to the VA was feasible when the angle formed by the VA and the subclavian artery (AVS) was $45^{\circ}$ or larger. In this study, even for a case with an AVS of $43^{\circ}$, which was sharpest, navigation of the 5MAX ACE to the VA was possible with a catheter exchange technique. This reason was considered to be attributable to the flexibility of the 5MAX ACE. However, if the AVS is too narrow, a transfemoral approach may be appropriate.

Some points need careful consideration when performing the DiBA technique. The soft tip of the 5MAX ACE could make insertion into an introducer sheath difficult. In such a case, using an attached inserter would facilitate smooth insertion of the 5MAX ACE into the introducer sheath.

When the 5MAX ACE becomes clogged with thrombus or when the techniques such as CAPTIVE, ${ }^{20}$ wherein the 5MAX ACE and stent retriever are removed as a single unit, are performed, the 5MAX ACE needs to be removed and again navigated to the BA. In that aspect, the DiBA technique, compared with the conventional guide catheter placement at the VA, would be disadvantageous. However, replacing the 5MAX ACE via the brachial artery appears to be easy.

In this study, the mean PTR time was longer and the rate of poor outcome was higher compared with the recommended PTR time ${ }^{11}$ and a recent report. ${ }^{12}$ One of these reasons could be that MET was performed for elderly patients with a high NIHSS. However, in all patients, the 5MAX ACE could be placed in the VA without difficulty, even via the left transbrachial approach. Additionally, $\mathrm{TICI}$ 2B or 3 recanalization was achieved, and there were no technical complications. It was considered that this technique would be useful especially for tortuous arteries in the elderly.

\section{CONCLUSION}

We described the DiBA technique, in which the Penumbra 5MAX ACE reperfusion catheter was used via the brachial artery as a guide catheter. In all patients, the 5MAX ACE could be easily navigated into the $\mathrm{VA}$, and $\mathrm{TICI} 2 \mathrm{~B}$ or 3 recanaliza- tion was achieved with no technical complications. It was considered to be useful, especially for tortuous arteries in the elderly. Further studies on the DiBA technique are needed.

\section{Fund}

None.

\section{Ethics Statement}

The Institutional Review Board approval was obtained at the Nagoya City University Graduate School of Medical Sciences in Nagoya, Japan.

\section{Conflicts of Interest}

The authors have no conflicts to disclose.

\section{REFERENCES}

1. Berkhemer OA, Fransen PS, Beumer D, van den Berg LA, Lingsma HF, Yoo AJ, et al. A randomized trial of intraarterial treatment for acute ischemic stroke. N Eng/ J Med 2015;372:11-20

2. Goyal M, Demchuk AM, Menon BK, Eesa M, Rempel JL, Thornton J, et al. Randomized assessment of rapid endovascular treatment of ischemic stroke. N Engl J Med 2015;372:1019-1030

3. Campbell BC, Mitchell PJ, Kleinig TJ, Dewey HM, Churilov L, Yassi $\mathrm{N}$, et al. Endovascular therapy for ischemic stroke with perfusion-imaging selection. N Engl J Med 2015;372:1009-1018

4. Saver JL, Goyal M, Bonafe A, Diener HC, Levy El, Pereira VM, et al. Stent-retriever thrombectomy after intravenous t-PA vs. t-PA alone in stroke. N Engl J Med 2015;372:2285-2295

5. Jovin TG, Chamorro A, Cobo E, de Miquel MA, Molina CA, Rovira A, et al. Thrombectomy within 8 hours after symptom onset in ischemic stroke. N Engl J Med 2015;372:2296-2306.

6. Saver JL, Goyal M, van der Lugt A, Menon BK, Majoie CB, Dippel $D W$, et al. Time to treatment with endovascular thrombectomy and outcomes from ischemic stroke: a meta-analysis. JAMA 2016;316:1279-1288

7. Giorgianni A, Biraschi F, Piano M, Mardighian D, Gasparotti R, Frigerio $\mathrm{M}$, et al. Endovascular treatment of acute basilar artery occlusion: Registro Endovascolare Lombardo Occlusione basilar artery (RELOBA) study group experience. J Stroke Cerebrovasc Dis 2018;27:2367-2374

8. Ribo M, Flores A, Rubiera M, Pagola J, Mendonca N, Rodriguez-Luna $D$, et al. Difficult catheter access to the occluded vessel during endovascular treatment of acute ischemic stroke is associated with worse clinical outcome. J Neurolnterv Surg 


\section{3;5 Supple 1:i70-i73}

9. Iwata T, Mori T, Miyazaki Y, Tanno Y, Kasakura S, Aoyagi Y. Anatomical features of the vertebral artery for transbrachial direct cannulation of a guiding catheter to perform coil embolization of cerebral aneurysms in the posterior cerebral circulation. Interv Neuroradiol 2015;21:381-386

10. Humphries W, Hoit D, Doss VT, Elijovich L, Frei D, Loy D, et al. Distal aspiration with retrievable stent assisted thrombectomy for the treatment of acute ischemic stroke. J Neurointerv Surg 2015;7:90-94

11. McTaggart RA, Ansari SA, Goyal M, Abruzzo TA, Albani B, Arthur $A J$, et al. Initial hospital management of patients with emergent large vessel occlusion (ELVO): report of the standards and guidelines committee of the Society of Neurolnterventional Surgery. J Neurointerv Surg 2017;9:316-323

12. Kaneko J, Ota T, Tagami T, Unemoto K, Shigeta K, Amano T, et al. Endovascular treatment of acute basilar artery occlusion: Tama-REgistry of Acute Thrombectomy (TREAT) study. J Neurol Sci 2019;401:29-33

13. Lee DG, Lee $D H$, Shim JH, Suh DC. Feasibility of the transradial or the transbrachial approach in various neurointerventional procedures. Neurointervention 2015;10:74-81

14. Ishikawa O, Tsutsumi K, Yoshikawa G, Saito A, Tsunoda S, Kondo E, et al. Thrombectomy using a method to directly insert an as- piration catheter into a 6-Fr sheath-introducer placed into the brachial artery. JNET 2018;12:456-462

15. Turk AS, Frei D, Fiorella D, Mocco J, Baxter B, Siddiqui A, et al. ADAPT FAST study: a direct aspiration first pass technique for acute stroke thrombectomy. J Neurointerv Surg 2014;6:260-264

16. Nguyen TN, Malisch T, Castonguay AC, Gupta R, Sun CH, Martin $\mathrm{CO}$, et al. Balloon guide catheter improves revascularization and clinical outcomes with the Solitaire device: analysis of the North American Solitaire Acute Stroke Registry. Stroke 2014;45:141145

17. Matsubara N, Miyachi S, Okamaoto T, Izumi T, Asai T, Yamanouchi T, et al. Spinal cord infarction is an unusual complication of intracranial neuroendovascular intervention. Interv Neuroradiol 2013;19:500-505

18. Agrawal A, Ziccardi MR, Witzke C, Palacios I, Rangaswami J. Cholesterol embolization syndrome: an under-recognized entity in cardiovascular interventions. J Interv Cardiol 2018;31:407-415

19. Oomura M, Yamada K, Anan C, Yamada G Hashimoto N, Kamimoto K. Transbrachial carotid artery stenting can prevent renal cholesterol embolism. Intern Med 2014;53:1017-1021

20. McTaggart RA, Tung EL, Yaghi S, Cutting SM, Hemendinger M, Gale HI, et al. Continuous aspiration prior to intracranial vascular embolectomy (CAPTIVE): a technique which improves outcomes. J Neurointerv Surg 2017;9:1154-1159 